\title{
A comparison of $E M$ and real-time PCR for the diagnosis of human polyomaviruses in urine from bone marrow transplant patients
}

\author{
S.S. Biel ${ }^{\text {a }}$, A. Nitsche ${ }^{\mathrm{a}, \mathrm{b}}$, A. Kurth ${ }^{\mathrm{a}}$, W. Siegert ${ }^{\mathrm{b}}$, M. Özel ${ }^{\mathrm{a}}$, H.R. Gelderblom ${ }^{\mathrm{a}}$ \\ a Robert Koch-Institut, 13353 Berlin, Germany \\ b Med. Klinik II, Charite - Campus Charite Mitte, 13353 Berlin, Germany
}

EM diagnosis of viral infections is more and more replaced by PCR assays, because the latter can generally be performed following routine laboratory protocols, while EM still depends greatly on the skill and experience of the personnel using the microscope. However, there is a continuing discussion whether PCR can be a satisfactory alternative to EM, and only few direct comparisons between PCR and EM diagnostic assays for one specific agent have been made.

In this study, the diagnostic performance of EM has been evaluated by (1) comparing different EM diagnostic preparation techniques (standard negative staining, ultracentrifugation prior to EM, immune-specific particle enrichment), and then (2) comparing the best of these EM assays with an upto-date PCR assay on a large number of clinical samples. Polyomavirus diagnosis from urine samples has been chosen, because neither EM nor PCR has been recognised as the best for this purpose.

The comparison of direct EM with UC and immuno enrichment showed that the sensitivity of EM diagnosis is improved by enrichment procedures. The comparison of UC/EM with quantitative realtime PCR on 571 clinical urine samples revealed an EM diagnostic sensitivity of $47 \%$ with a specificity of $100 \%$. Moreover, a linear relationship between viral genome concentration and the proportion of urine samples positive by EM has been detected, showing a $50 \%$ probability for a positive EM diagnosis for urine samples with $10^{6}$ viral genome equivalents per millilitre (ge $\left./ \mathrm{mL}\right)$; this probability is $0 \%$ only for urine samples below $10^{3} \mathrm{ge} / \mathrm{mL}$, and at $10^{9} \mathrm{ge} / \mathrm{mL}$ it is about $100 \%$. We conclude that UC/ EM should be used as the first diagnostic tool for the detection of polyomavirus in urine, since it has proven to be a fast (15 min until positive diagnosis) and reliable technique (positive predictive value $100 \%$ ). Afterwards, PCR should be applied to EM negative urine samples to reduce the risk of false negative results. 


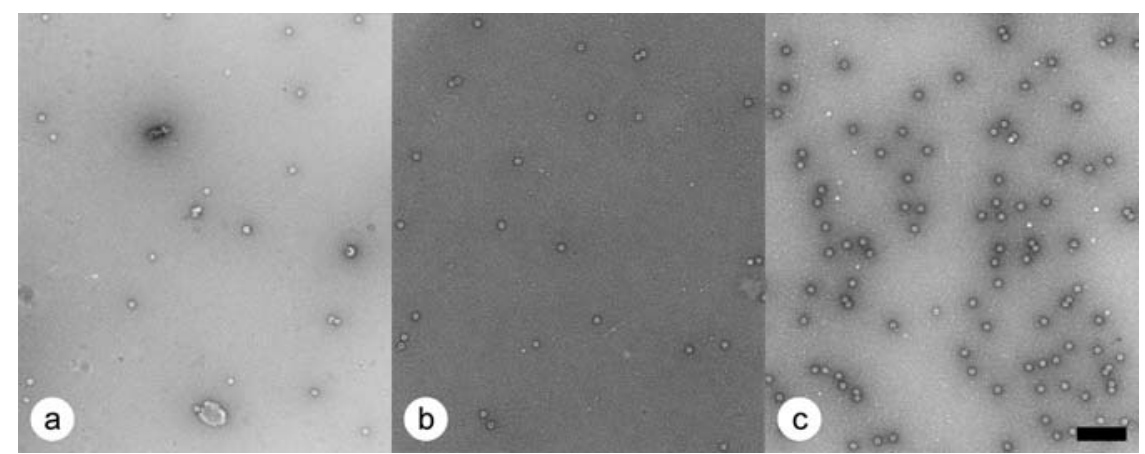

Figure 1: Comparison of direct EM with enrichment by UC and immuno-EM (SPIEM)

Polyomavirus particles from a fresh undiluted urine sample bound to the EM grid after (a) no pretreatment; (b) ultracentrifugation for $60 \mathrm{~min}$ at 100,000 $\mathrm{g}$ and resuspension of the sediment in $50 \mu \mathrm{L}$ double-distilled water; (c) antibody-mediated enrichment to the grid by SPIEM. All samples were negatively stained with $2 \%(\mathrm{w} / \mathrm{v})$ uranyl acetate prior to EM examination. Bar: $250 \mathrm{~nm}$

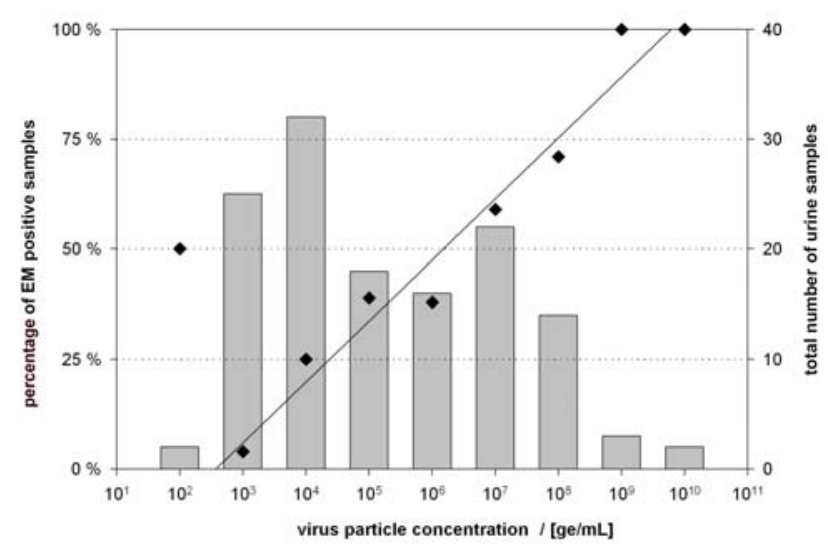

Figure 2: Correlation between number of particles and probability for positive EM diagnosis

All urine samples positive in quantitative polyomavirus PCR were grouped depending on their viral load in decades from $10^{2} \mathrm{ge} / \mathrm{mL}$ to $10^{10} \mathrm{ge} / \mathrm{mL}$ (total number within each decade is shown as grey columns). The percentage of EM-positive samples within each of these groups is drawn as black diamonds. Linear regression of the data reveals a direct linear correlation between the viral load and the proportion of urine samples positive after EM, as shown by the straight line $\left(r^{2}=0.9\right)$. 